\title{
Mesenchymal Stem Cells as Vectors for Lung Cancer Therapy
}

\author{
Krishna K. Kolluri $^{\mathrm{a}}$ Geoff J. Laurent ${ }^{\mathrm{b}}$ Sam M. Janes ${ }^{\mathrm{a}}$ \\ ${ }^{a}$ Lungs for Living Research Centre, University College London, London, UK; ${ }^{b}$ Centre for Cell Therapy and \\ Regenerative Medicine, University of Western Australia, Perth, W.A., Australia
}

\section{Key Words}

Apoptosis · Delivery vectors · Homing · Lung cancer · Mesenchymal stem cells $\cdot$ TRAIL

\begin{abstract}
Despite recent advances in treatment, lung cancer accounts for one third of all cancer-related deaths, underlining the need of development of new therapies. Mesenchymal stem cells (MSCs) possess the ability to specifically home into tumours and their metastases. This property of MSCs could be exploited for the delivery of various anti-tumour agents directly into tumours. However, MSCs are not simple delivery vehicles but cells with active physiological process. This review outlines various agents which can be delivered by MSCs with substantial emphasis on TRAIL (tumour necrosis factorrelated apoptosis-inducing ligand).
\end{abstract}

Copyright $\odot 2013$ S. Karger AG, Basel

Previous articles in this series: 1. Bouros D, Laurent G: Regenerative medicine and stem cells - Prometheus revisited. Respiration 2013;85:1-2. 2. Kolios G, Moodley Y: Introduction to stem cells and regenerative medicine. Respiration 2013;85:3-10. 3. Ardhanareeswaran K, Mirotsou M: Lung stem and progenitor cells. Respiration 2013;85:89-95. 4. Tzouvelekis A, Ntolios P, Bouros D: Stem cell treatment for chronic lung diseases. Respiration 2013;85:179192. 5. Cárdenes N, Cáceres E, Romagnoli M, Rojas M: Mesenchymal stem cells: a promising therapy for the acute respiratory distress syndrome. Respiration 2013;85:267-278. 6. Farkas L, Kolb M: Vascular repair regeneration as a therapeutic target for pulmonary arterial hypertension. Respiration 2013;85:355-364.

\section{KARGER}

E-Mail karger@karger.com www.karger.com/res
(C) 2013 S. Karger AG, Basel 0025-7931/13/0856-0443\$0/0

Karger Open access

This is an Open Access article licensed under the terms of the Creative Commons Attribution-NonCommercial 3.0 License (www.karger.com/OA-license-WT), applicable to the online version of the article only. Distribution for noncommercial purposes only.

\section{Introduction}

Lung cancer is one of the leading causes of mortality and morbidity in the world and accounts for one third of all the cancer-related deaths [1]. An estimated 1.61 million people across the world were diagnosed with lung cancer in 2008 [2]. Non-small cell lung cancer accounts for $80 \%$ of all the lung cancer cases and its 5 -year survival remains $8-15 \%$ [3]. Current treatments of lung cancer include surgery, radiotherapy and chemotherapy. For metastatic lung cancer, chemotherapy with the combination of cisplatin and pemetrexed is used as first-line treatment. EGFR antagonists like erlotinib and gefitinib are recommended in the low percentage of cancers with EGFR-tyrosine kinase mutations. Despite the introduction of new therapies, lung cancer kills more people than breast, colon and prostate cancers combined, and there has been little overall improvement in patient survival in 3 decades [4]. This justifies the need for new and innovative therapies. Stem cells may be able to deliver such therapies to the site of tumours with minimal adverse effects.

\section{Mesenchymal Stem Cells}

Mesenchymal stem cells (MSCs) are a type of bone marrow-derived stem cell, which can differentiate in vitro into osteoblasts, chondrocytes and adipocytes. They do not possess any unique markers for their identification, so their identification relies on the expression of CD73,

Dr. Sam M. Janes, MSc, PhD, FRCP

Lungs for Living Research Centre, University College London

5 University Street

London WC1E 6JJ (UK)

E-Mail s.janes@ucl.ac.uk 
CD90 and CD105 while lacking CD34, CD45 and other haematopoietic stem cell markers [5].

MSCs lack the expression of MHC II and its co-stimulatory molecules CD80 and CD86 and CD40 [6]. This low immunogenicity of MSCs may make allogeneic cells incapable of eliciting an immune response when used in immunocompetent patients hence avoiding the need for human leucocyte antigen matching and allowing an offthe-shelf therapy [7]. This paves the way for using MSCs as cell-based therapeutic vectors for the treatment of cancers. Indeed, clinical trials using MSCs for treatment of a wide variety of diseases including graft-versus-host disease and Crohn's disease have proved delivery of allogeneic MSCs is safe. MSCs are also easily extracted and readily expandable with up to 50 population doublings in 10 weeks [8]. Taken together, these properties may enable the creation of MSC cell banks.

\section{MSC Homing to Tumours and Mediators Involved}

It has been widely demonstrated that MSCs home to and infiltrate into areas of new stroma formation possibly forming crucial stromal support [9]. This has been shown in several models, including lung metastases [10, 11], Kaposi sarcomas [12] and gliomas [13]. However, their role once integrated within the tumour environment is unknown.

The precise mechanism of homing of MSCs to the tumours is not fully mapped, but it was widely accepted that the chemokines released by the tumours attract MSCs. This is substantiated by the presence of a wide variety of chemokine receptors on the MSC cell surface and experiments in vitro and in mouse models that have either over- or under-expressed these receptors, showing a change in MSC homing capabilities [14-18]. There are several different ligands and receptors postulated to play a role in MSC migration. However, there is general agreement that these studies have not yet been able to pinpoint the exact chemokine and its respective receptor that governs MSC tumour tropism, and there may indeed be a combination of receptors and chemokines responsible.

CXCL12 and its receptor CXCR4 have generated particular interest in MSC homing. Their knockouts are universally fatal in utero and their role in migration of haematopoietic cell migration is well characterised $[19,20]$. Several tumours are known to release CXCL12 [21, 22] and studies show over-expression of these receptors leads to increased MSCs migration to infarcted myocardium [23]. However, knockdown of these receptors does not mitigate MSC homing capability [24]. This can be interpreted that the CXCL12 ligand and its receptor CXCR4 might be capable of inducing some MSC migration but they are not the only receptors responsible for MSC homing. This is further substantiated by the fact that some MSCs do not express this receptor at all [18].

Work on MSC homing is complicated and varying results may be explained by a number of factors. MSCs are extracted from various tissues and their lack of unique identification markers to classify them results in a number of different populations being used making cross-referencing results difficult. Furthermore, different in vitro culture conditions and the passage numbers used alter the expression of cell surface receptors $[16,25]$. This results in lack of homogeneity of MSCs being used in laboratories likely explaining the variability seen. Taken together, MSC migration is highly likely to be dependent on the expression of a number of chemokine receptors on their cell surface $[26,27]$.

\section{MSCs as Delivery Vectors for Pro-Apoptotic Agents}

The homing capability of MSC can be exploited to deliver pro-apoptotic agents straight into the tumour micro-environment. Several studies have achieved this with varying success and are summarised in table 1.

The majority of studies have used MSCs engineered to express and deliver a variety of cytokines. Interleukin (IL)2 , an immune modulatory cytokine, has been shown when over-expressed by MSCs to improve immune surveillance against tumours and reduce metastasis from a subcutaneous model [28]. Similarly CX3CL1, a chemokine which activates both T cells and NK cells when delivered by MSCs, leads to a substantial decrease in lung tumours induced by intravenous delivery of melanoma cells [29]. Interferon- $\beta$, which induces differentiation and S-phase accumulation leading to apoptosis, when expressed by genetically engineered MSCs suppresses pancreatic tumours, prostate cancers, breast cancers and melanomas in animal models [30-33]. Finally, a similar effect occurs with the delivery of IL-12-expressing MSC in renal cell carcinoma [34].

An exciting set of viruses which selectively target and inhibit tumour cells without affecting normal cells are termed oncolytic viruses [35]. These viruses are genetically engineered to selectively infect and destroy tumour cells. However, their delivery to the tumour site remains a major challenge [36]. Using MSC tumour tropism raises a new possible modality of virus delivery. MSCs would again act as carrier vectors for the oncolytic virus and this 
Table 1. Anti-tumour agents delivered by MSCs

\begin{tabular}{|c|c|c|c|}
\hline Agent & Rationale & Model & References \\
\hline IL-2 & immune modulatory & subcutaneous model & 28 \\
\hline Interferon- $\beta$ & induces differentiation and S-phase arrest & $\begin{array}{l}\text { pancreatic cancer } \\
\text { prostate cancer } \\
\text { breast cancer } \\
\text { melanoma }\end{array}$ & $30-34$ \\
\hline IL-12 & activates T cells and NK cells & renal cell carcinoma & 34 \\
\hline Oncolytic virus & destroys tumours by viral infection & $\begin{array}{l}\text { breast cancer } \\
\text { lung cancer } \\
\text { ovarian cancer } \\
\text { lung metastasis }\end{array}$ & $37-39$ \\
\hline $\mathrm{rCE}$ & $\begin{array}{l}\text { converts the pro-drug CPT- } 11 \text { to } \mathrm{SN}-38 \text {, } \\
\text { a potent topo-isomerase I inhibitor }\end{array}$ & glioma & 44 \\
\hline Nanoparticle & silica nanorattle-doxorubicin & glioma & 47 \\
\hline TRAIL & tumour-specific death ligand & $\begin{array}{l}\text { glioma } \\
\text { pancreatic cancer } \\
\text { lung metastasis }\end{array}$ & $10,54,55$ \\
\hline
\end{tabular}

MSCs have been used to deliver a variety of anti-tumour agents. The rationale behind their use and the models used are described with references. HSV-tk = Herpes simplex virus thymidine kinase; rCE = rabbit carboxylesterase enzyme.

delivery mechanism comes with the added advantage of limiting the recipient immune response to the virus to a minimum. This technique has been very successfully used in several tumour models, which include breast and lung metastases [37, 38] and ovarian cancer [39]. Indeed, a recent study has demonstrated the feasibility of treating ovarian cancer using MSC oncolytic virus, paving the way for a phase I clinical trial [40].

MSCs have also been engineered to express an enzyme which converts a pro-drug into a cytotoxic agent at the site of tumours. This has been successfully demonstrated in a glioma model [41] where MSCs were engineered to express the herpes simplex virus-thymidine kinase which converts the prodrug ganciclovir at the tumour site. However, this approach may be limited by the toxicity to the carrier MSCs. A similar approach has been used to convert 5-fluorocytosine to 5-fluorouracil by MSCs expressing cytosine deaminase enzyme in melanoma [42] and colon cancer models [43]. MSCs have also been genetically modified to express rabbit carboxylesterase enzyme, which can efficiently convert the prodrug CPT-11 into the active drug $\mathrm{SN}-38$, which acts as a potent topoisomerase I inhibitor [44]. In a different approach, nano-sized exosomes which are mass produced by MSC [45] have been extracted and used to deliver a variety of therapeutics including siRNA [46].

There is increasing interest in the use of nanoparticles in a variety of biomedical applications. However, the ability to deliver them efficiently to a disease that is systemically distributed remains a key challenge. Again, MSC tumour tropism has been used as a method of tumour targeting. A silica nanorattle-doxorubicin drug delivery system was efficiently anchored to MSCs by specific antibody targeting the CD90 receptor on the MSC cell surface and successfully delivered into a glioma model [47]. Interest has also arisen in using nanoparticles as a method of tracking MSC homing to tumours. Iron oxide nanoparticles phagocytized by MSCs have been used to identify MSC homing to pulmonary lung metastases using magnetic resonance imaging [11]. 


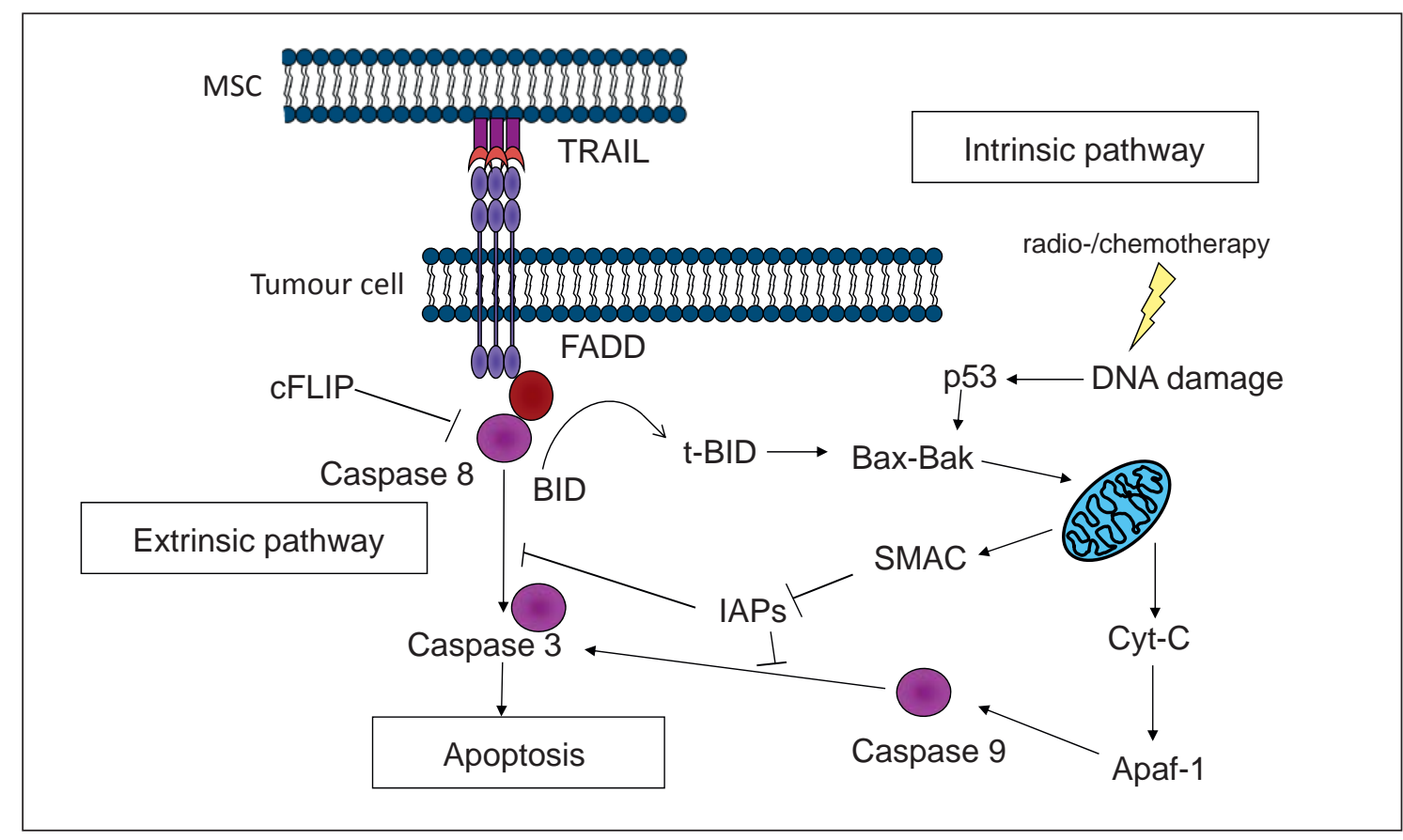

Fig. 1. TRAIL signalling induces the extrinsic apoptotic pathway. TRAIL triggers the extrinsic apoptotic pathway while conventional chemotherapeutics and radiotherapy trigger the intrinsic apoptotic pathway mediated by mitochondria. There is crosstalk between the two pathways mediated by cleavage of BID into t-BID by caspase 8. cFLIP and IAPs are potent inhibitors of apoptotic proteins and their inhibition could induce synergistic effects by simultaneous triggering of both pathways. FADD $=$ FAS-activated death domain; $\mathrm{BID}=\mathrm{BH} 3$ interactingdomain death agonist; $\mathrm{BAK}=\mathrm{Bcl}-2$ homologous antagonist; $\mathrm{Cyt}-\mathrm{C}=$ cytochrome $\mathrm{c}$; Apaf-1 = apoptotic proteaseactivating factor 1 .

\section{Delivery of TRAIL}

TRAIL (tumour necrosis factor-related apoptosis-inducing ligand) is the most studied and well-characterised pro-apoptotic agent widely accepted to be ideal as MSC cargo. TRAIL, also known as APO2 ligand, is a type II transmembrane protein with 281 amino acids and a member of the TNF death ligand superfamily. TRAIL triggers the extrinsic death pathway (fig. 1). The physiological function of TRAIL is not fully understood. However, it is believed to play a role in the control of autoreactive immune cells and immune surveillance especially against transformed cells [48].

There are five types of TRAIL receptors identified to date and they include TRAIL-R1, TRAIL-R2, TRAIL-R3 and TRAIL-R4. However, only TRAIL-R1 (death receptor-4, DR-4) and TRAIL-R2 (DR-5) are able to transduce a signal into the cell after binding of TRAIL to their extracellular domains. The receptors TRAIL-R3 and TRAIL-R4 do possess extracellular domains capable of binding to the ligand but lack an intracellular cytoplasmic domain, thus failing to mediate death signals to the intracellular apoptotic machinery. Hence, TRAIL-R3 and TRAIL-R4 act as decoy receptors that antagonize TRAILinduced apoptosis. All these receptors form heterotrimers upon binding of the ligand. Osteoprotegerin is a soluble protein which possesses the capability of binding to TRAIL with low affinity [49]. This protein is not expressed on the cell surface.

The novelty of TRAIL is that it only induces apoptosis in transformed cells with virtually no effect on normal cells. This makes TRAIL a unique therapeutic with very few off-target adverse effects characteristic of chemotherapeutic agents and radiation. The mechanism for this selective targeting of tumour cells is not well characterised. The decoy receptor theory states that the normal cells express decoy receptors while the transformation of cells makes them express DR4 and DR5 thus making them vulnerable to TRAIL [50]. However, this theory is not widely accepted and it is believed that the selective cytotoxicity of TRAIL occurs beneath the cell membrane. One intriguing study shows that in tumours TRAIL receptors 
lead to apoptosis when expressed within lipid rafts of the cell membrane (fig. 2). These rafts concentrate pro-apoptotic downstream signalling molecules internally. In normal tissue, however, the receptors are largely in the nonraft areas and TRAIL binding can lead to pro-survival pathway activation [51]. Other studies have identified the TRAIL receptor glycosylation status [52] and pre-ligand binding assembly domain of receptors playing roles in TRAIL sensitivity [53]. It is likely that TRAIL sensitivity is multi-factorial and cannot be ascribed to any single mechanism.

The selective tumour-specific cytotoxicity of TRAIL has led to hailing it as a 'silver bullet' for the treatment of cancer. However, its limited bioavailability and poor pharmacokinetic profile have made its use a serious challenge. The half-life of TRAIL is very short at around 30 min [54]. To circumvent this problem, we and others have engineered MSC to constitutively express TRAIL. This has been demonstrated effective in several models, including glioma [55], pancreatic cancer [56] and a lung metastasis model [43]. MSC-TRAIL cells home into the tumours and expresses TRAIL leading to selective apoptosis of tumour cells with no detectable cytotoxicity to the surrounding tissue. We have used a Tet-On promoter system allowing the controlled release of TRAIL with the addition of doxycycline [10]. Interestingly, the tumour killing capability of MSCs expressing TRAIL is significantly higher than that of recombinant TRAIL [10].

However, not all tumours are fully sensitive to TRAIL. TRAIL triggers the extrinsic apoptotic pathway, while conventional chemotherapeutics and radiation trigger the intrinsic apoptotic pathway (fig. 2). It would be ideal to trigger the simultaneous activation of both pathways to harness synergistic effects. There is known synergy between traditional chemotherapy agents and TRAIL. This synergy results in increased apoptosis by amplification of apoptotic signals through crosstalk between the two apoptotic pathways [57]. A number of chemotherapeutic agents have demonstrated synergy both in vitro and in vivo: cisplatin [58], vorinostat [59], pemetrexed [60], sunitinib [61], etoposide [62], doxorubicin [62] and bortezomib [63]. Furthermore, the combination of chemotherapeutics and MSC expressing TRAIL was shown to be synergistic with bortezomib [64] in myeloma cells and vorinostat in lung cancer [65].

Identifying and targeting proteins responsible for TRAIL resistance may also increase the anti-tumour potency of TRAIL, such as cFLIP (cellular FLICE-inhibitory protein) [66, 67], cIAP1/cIAP2 [68] and XIAP [56, 69, 70]. Another potential use of MSC-delivered TRAIL

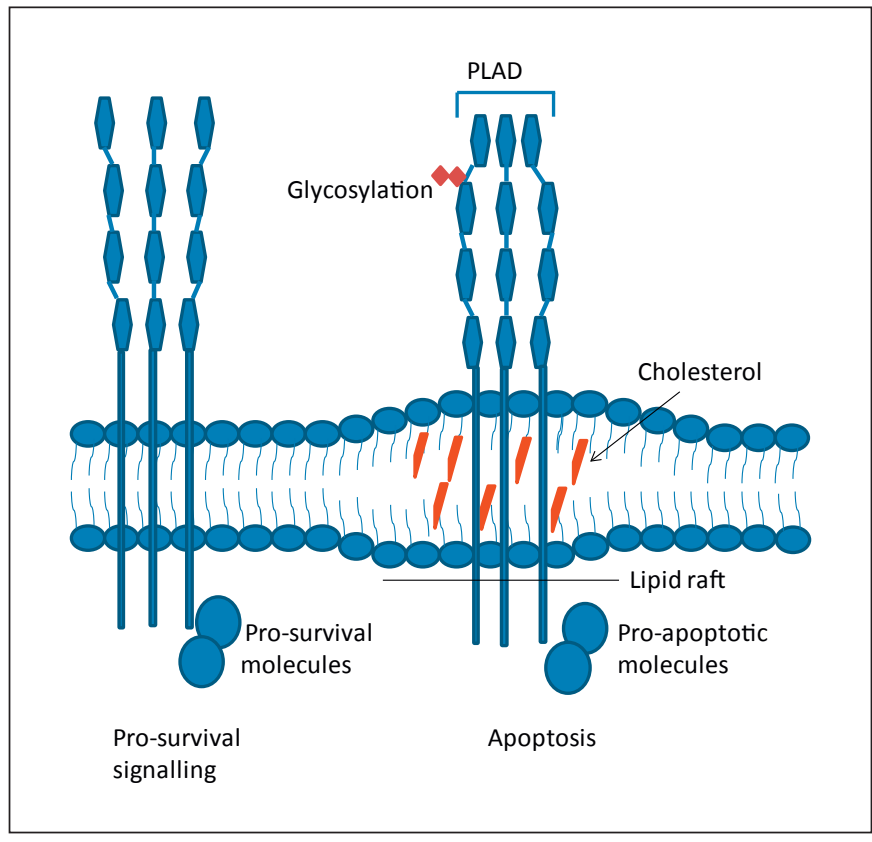

Fig. 2. TRAIL signalling varies based on death receptor location and glycosylation. TRAIL induces apoptosis pathways when the death receptors are glycosylated or forms pre-ligand binding assembly domain (PLAD) or when located on lipid rafts.

would be to decrease the required dosage of chemotherapeutic agents improving drug tolerance and reducing adverse effects [71].

\section{Immunosuppressive Effects of MSCs}

The ability of MSCs to home effectively to tumours makes them an attractive therapeutic option. However, MSCs are not merely vehicles which transport the therapies but are cells possessing physiological properties.

It is widely accepted that in large numbers MSCs possess immunosuppressive effects in vitro. They are capable of arresting the immune cells in G0/G1 phases thus preventing the S-phase entry and subsequent cell division. This has been demonstrated in T cells [72], B cells [73] and dendritic cells [74]. This leads to reduced cytotoxic capability of $\mathrm{T}$ cells and antibody production of $\mathrm{B}$ cells. MSCs also exert an immunosuppressive effect by activation of regulatory T cells. These properties of MSCs are clinically exploited for the treatment of graft-versus-host disease after bone marrow transplantation [75].

These anti-inflammatory effects have been tested in a number of clinical trials in inflammatory conditions, such 


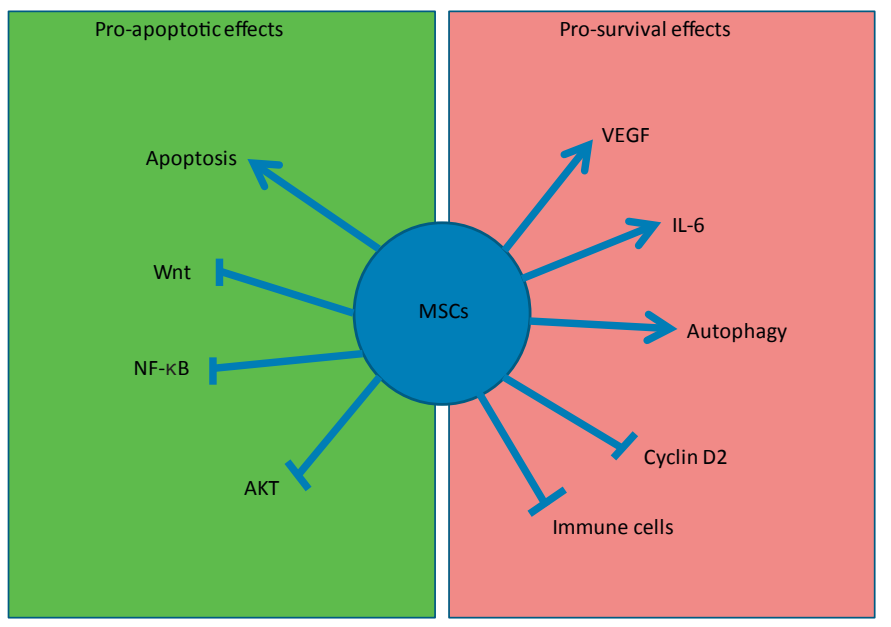

Fig. 3. MSCs are not inert carriers. MSCs exert both pro-survival and pro-apoptotic effects on tumours. Their pro-apoptotic effects include inhibition of Akt, Wnt and NF- $\kappa$ B signalling. MSCs themselves induce apoptosis in some tumours. They also exert pro-survival effects by inducing VEGF and STAT3 activation. They suppress immune cells thereby reducing immune surveillance of tumours. They undergo autophagy and release pro-survival paracrine factors. They inhibit cyclin D2.

as inflammatory bowel disease and chronic obstructive pulmonary disease, and in other diseases, such as cardiac disease. Several studies show an enhanced cardiac function [76-79] and reduced infarct size [78] by injecting MSCs after myocardial infarction and chronic ischemic heart failure. The exact mechanisms of this effect are to be characterised but have been attributed to anti-inflammatory properties of MSCs. These anti-inflammatory properties have also been demonstrated in murine models of pulmonary fibrosis and acute lung injury, and were thought the result of the paracrine effect of secreted tropic factors [80]. The Battacharya laboratory has also shown that MSCs can protect against acute lung injury by donating their mitochondria to alveolar epithelial cells [81].

The immunosuppressive capability of MSCs could, however, be a double-edged sword. Their immunosuppressive nature could potentially interfere with any physiological anti-cancer immune cell function in the tumour environment.

\section{Direct Effects of MSCs on Tumour Biology}

Reports on untransduced MSC effects on tumour growth are mixed. The majority of work suggests MSCs not only home to tumours but also have intrinsic anti- tumour properties. MSCs alone lead to benefit in a murine glioma model [82], in our studies of pulmonary metastases [6] and in a breast cancer metastasis model with either intravenous or intra-tumour delivery of MSCs significantly reducing the growth and metastasis [83]. The mechanism of this anti-tumour effect is not fully established, although MSCs have been shown to down-regulate many pro-survival genes, such as AKT in the Kaposi sarcoma mouse model [12] and NF- $\kappa B$ in hepatoma and breast cancer cells [84].

In specific context, however, MSCs can appear to be tumour promoting, which was demonstrated by tumour development after subcutaneous co-administration of MSCs with allogeneic melanoma cells producing tumours, while allogeneic melanoma cells seeded on their own are not capable of tumour induction [85]. This effect was attributed to the immunosuppressive effect of MSCs which suppressed the host immune reaction to the allogeneic melanoma cells.

As discussed earlier, MSCs do produce a wide array of chemokines, cytokines and growth factors (fig. 3). They may also produce and secrete growth factor signalling that promotes survival in tumour cells resulting in enhanced tumour burden and metastases. It has been demonstrated that MSCs enhance the in vivo growth of Burkett's lymphoma cells through a VEGF-dependent mechanism [86]. The growth of breast cancer cells was augmented by IL- 6 secreted by MSCs via STAT3 activation [87, 88]. It has also been shown that MSCs can down-regulate cyclin D2 and arrest chronic myeloid leukaemia cells in G0/G1 phase preserving their proliferative capacity and reducing apoptosis in vivo [74]. Furthermore, under the nutrient-depleted conditions of the tumour micro-environment, MSCs utilize autophagy for survival and secrete anti-apoptotic factors that facilitate solid tumour survival and growth in breast cancer cells [89].

Another important and serious concern is the ambiguity that MSCs might themselves undergo malignant transformation. Karyotype abnormalities have been noticed after in vitro passage of murine MSCs [90-92] and transformations of bone marrow-derived cells have been implicated in a murine gastric carcinoma model [93]. Human MSCs, however, have stable karyotypes in culture and exhibit senescence with features of shortening telomeres over a 44-week culture period [94]. There have been about 300 clinical trials in clinicaltrials.gov injecting MSCs for cell therapy with no reported incident of MSC malignant transformation. 
Developing a cellular therapy using MSCs as delivery vectors is the ultimate goal of this area of research and MSCs have exhibited the potential for clinical translation. MSCs can be easily isolated, cultured in flasks and genetically modified. Their allogeneic application confers them an added advantage of being a possible off-the-shelf therapeutic. Their ability to target metastasis and provide a local high concentration of their cargo makes them unique.

Many phase I and II clinical trials involving MSCs for a variety of treatments were recorded in the largest clinical trial database, clinicaltrials.gov. The therapeutic areas include graft-versus-host disease, ischaemic cardiac disease, Crohn's disease and chronic obstructive pulmonary disease [95]. However, there are no reported trials of the use of MSC as delivery agents for anti-tumour therapy to date.

\section{Conclusion}

MSC have the potential to be ideal delivery vectors for a variety of pro-apoptotic agents in treating cancers. The lack of knowledge of MSC physiology within the tumour environment is producing caution, and more robust studies characterising their homing mechanisms may improve proposed therapies. Indeed, simple questions, such as how many cells need to be given and when, remain unanswered. However, their role as an adjunct in patients with metastatic tumours looks hopeful.

\section{Acknowledgement}

Dr. Janes is a Wellcome Trust Senior Research Fellow in Clinical Science.

\section{Financial Disclosure and Conflicts of Interest}

All authors declare no conflicts of interest.

\section{References}

$\checkmark 1$ Jemal A, et al: Cancer statistics, 2007. CA Cancer J Clin 2007;57:43-66.

- 2 Ferlay J, Shin HR, Bray F, et al: Globocan 2008: Cancer Incidence and Mortality Worldwide: IARC CancerBase No. 10. Lyon, International Agency for Research on Cancer, 2010. http://globocan.iarc.fr.

-3 Jemal A, et al: Cancer statistics, 2005. CA Cancer J Clin 2005;55:259-259.

4 Crino L, Foglietta J, Hamzaj A: Development of new first-line therapeutic options for nonsmall-cell lung cancer. Lung Cancer 2006; 54:S19-S24.

5 Dominici M, et al: Minimal criteria for defining multipotent mesenchymal stromal cells. The International Society for Cellular Therapy position statement. Cytotherapy 2006;8 315-317.

-6 Javazon EH, Beggs KJ, Flake AW: Mesenchymal stem cells: paradoxes of passaging. Exp Hematol 2004;32:414-425.

7 Weiss DJ: Stem cells and cell therapies for cystic fibrosis and other lung diseases. Pulm Pharmacol Ther 2008;21:588-594.

8 Giordano A, Galderisi U, Marino IR: From the laboratory bench to the patients bedside: an update on clinical trials with mesenchymal stem cells. J Cell Physiol 2007;211:27-35.

9 Bonnet D: Biology of human bone marrow stem cells. Clin Exp Med 2003;3:140-149.

10 Loebinger MR, et al: Mesenchymal stem cell delivery of TRAIL can eliminate metastatic cancer. Cancer Res 2009;69:4134-4142.
11 Loebinger MR, et al: Magnetic resonance imaging of mesenchymal stem cells homing to pulmonary metastases using biocompatible magnetic nanoparticles. Cancer Res 2009;69: 8862-8867.

12 Khakoo AY, et al: Human mesenchymal stem cells exert potent antitumorigenic effects in a model of Kaposi's sarcoma. J Exp Med 2006 203:1235-1247.

13 Nakamizo A, et al: Human bone marrow-derived mesenchymal stem cells in the treatment of gliomas. Cancer Res 2005;65:33073318.

14 Lopez Ponte A, et al: The in vitro migration capacity of human bone marrow mesenchymal stem cells: comparison of chemokine and growth factor chemotactic activities. Stem Cells 2007;25:1737-1745.

15 Ringe J, et al: Towards in situ tissue repair: human mesenchymal stem cells express chemokine receptors CXCR1, CXCR2 and CCR2 and migrate upon stimulation with CXCL8 but not CCL2. J Cell Biochem 2007;101:135146.

16 Honczarenko M, et al: Human bone marrow stromal cells express a distinct set of biologically functional chemokine receptors. Stem Cells 2006;24:1030-1041.

17 Sordi V, et al: Bone marrow mesenchymal stem cells express a restricted set of functionally active chemokine receptors capable of promoting migration to pancreatic islets. Blood 2005;106:419-427.
18 Von Luttichau I, et al: Human adult CD34(-) progenitor cells functionally express the chemokine receptors CCR1, CCR4, CCR7, CXCR5, and CCR10 but not CXCR4. Stem Cells Dev 2005;14:329-336.

19 Phillips RJ, et al: Circulating fibrocytes traffic to the lungs in response to CXCL12 and mediate fibrosis. J Clin Invest 2004;114:438-446.

$20 \mathrm{Xu} \mathrm{J}$, et al: Role of the SDF-1/CXCR4 axis in the pathogenesis of lung injury and fibrosis. Am J Respir Cell Mol Biol 2007;37:291-299.

21 Dwyer RM, et al: Monocyte chemotactic protein-1 secreted by primary breast tumors stimulates migration of mesenchymal stem cells. Clin Cancer Res 2007;13:5020-5027.

22 Orimo A, et al: Stromal fibroblasts present in invasive human breast carcinomas promote tumor growth and angiogenesis through elevated SDF-1/CXCL12 secretion. Cell 2005; 121:335-348.

23 Cheng Z, et al: Targeted migration of mesenchymal stem cells modified with CXCR4 gene to infarcted myocardium improves cardiac performance. Mol Ther 2008;16:571-579.

24 Ip JE, et al: Mesenchymal stem cells use integrin beta 1 not CXC chemokine receptor 4 for myocardial migration and engraftment. Mol Biol Cell 2007;18:2873-2882.

25 Rombouts WJC, Ploemacher RE: Primary murine MSC show highly efficient homing to the bone marrow but lose homing ability following culture. Leukemia 2003;17:160-170. 
26 Chamberlain G, Fox J, Ashton B, et al: Concise review: mesenchymal stem cells: their phenotype, differentiation capacity, immunological features, and potential for homing. Stem Cells 2007;25:2739-2749.

27 Ozaki Y, et al: Comprehensive analysis of chemotactic factors for bone marrow mesenchymal stem cells. Stem Cells Dev 2007;16:119129.

28 Chen X, et al: A tumor-selective biotherapy with prolonged impact on established metastases based on cytokine gene-engineered MSCs. Mol Ther 2008;16:749-756.

29 Xin H, et al: Targeted delivery of CX3CL1 to multiple lung tumors by mesenchymal stem cells. Stem Cells 2007;25:1618-1626.

- 30 Kidd S, et al: Mesenchymal stromal cells alone or expressing interferon-beta suppress pancreatic tumors in vivo, an effect countered by anti-inflammatory treatment. Cytotherapy 2010;12:615-625.

- 31 Studeny M, et al: Bone marrow-derived mesenchymal stem cells as vehicles for interferonbeta delivery into tumors. Cancer Res 2002; 62:3603-3608.

-32 Studeny M, et al: Mesenchymal stem cells: potential precursors for tumor stroma and targeted-delivery vehicles for anticancer agents. J Natl Cancer Inst 2004;96:1593-1603.

-33 Ren C, Kumar S, Chanda D, et al: Cancer gene therapy using mesenchymal stem cells expressing interferon-beta in a mouse prostate cancer lung metastasis model. Gene Ther 2008;15:1446-1453.

34 Gao P, et al: Therapeutic potential of human mesenchymal stem cells producing IL-12 in a mouse xenograft model of renal cell carcinoma. Cancer Lett 2010;290:157-166.

- 35 Russell SJ, Peng KW: Viruses as anticancer drugs. Trends Pharmacol Sci 2007;28:326333.

- 36 Russell SJ, Peng K-W, Bell JC: Oncolytic virotherapy. Nat Biotechnol 2012;30:658-670.

37 Hakkarainen T, et al: Human mesenchymal stem cells lack tumor tropism but enhance the antitumor activity of oncolytic adenoviruses in orthotopic lung and breast tumors. Hum Gene Ther 2007;18:627-641.

- 38 Stoff-Khalili MA, Rivera AA, Mathis JM, et al: Mesenchymal stem cells as a vehicle for targeted delivery of CRAds to lung metastases of breast carcinoma. Breast Cancer Res Treat 2007;105:157-167.

-39 Komarova S, et al: Mesenchymal progenitor cells as cellular vehicles for delivery of oncolytic adenoviruses. Mol Cancer Ther 2006;5: 755-766.

40 Mader EK, Butler G, Dowdy SC, et al: Optimizing patient derived mesenchymal stem cells as virus carriers for a phase I clinical trial in ovarian cancer. J Transl Med 2013;11:20.

-41 Uchibori R, et al: Retroviral vector-producing mesenchymal stem cells for targeted suicide cancer gene therapy. J Gene Med 2009;11: 373-381.
42 Kucerova L, Matuskova M, Pastorakova A, et al: Cytosine deaminase expressing human mesenchymal stem cells mediated tumour regression in melanoma bearing mice. J Gene Med 2008;10:1071-1082.

43 Kucerova L, et al: Adipose tissue-derived human mesenchymal stem cells mediated prodrug cancer gene therapy. Cancer Res 2007; 67:6304-6313.

44 Choi SA, et al: Human adipose tissue-derived mesenchymal stem cells: characteristics and therapeutic potential as cellular vehicles for prodrug gene therapy against brainstem gliomas. Eur J Cancer 2011;48:129-137.

45 Yeo RWY, et al: Mesenchymal stem cell: an efficient mass producer of exosomes for drug delivery. Adv Drug Deliv Rev 2013;65:336-341.

46 Alvarez-Erviti L, et al: Delivery of siRNA to the brain by systemic injection of targeted exosomes. Hum Gene Ther 2011;22:A42A42.

47 Li L, et al: Silica nanorattle-doxorubicin-anchored mesenchymal stem cells for tumortropic therapy. Acs Nano 2011;5:7462-7470.

48 LeBlanc HN, Ashkenazi A: Apo2L/TRAIL and its death and decoy receptors. Cell Death Differ 2003;10:66-75.

49 Mahalingam D, et al: TRAIL receptor signalling and modulation: are we on the right TRAIL? Cancer Treat Rev 2009;35:280-288.

50 Riccioni R, Pasquini L, Mariani G, et al: TRAIL decoy receptors mediate resistance of acute myeloid leukemia cells to TRAIL. Haematologica 2005;90:612-624.

51 Song JH, et al: Lipid rafts and nonrafts mediate tumor necrosis factor-related apoptosisinducing ligand-induced apoptotic and nonapoptotic signals in non-small cell lung carcinoma cells. Cancer Res 2007;67:6946-6955.

52 Wagner KW, Punnoose EA, Januario T et al: Death-receptor $O$-glycosylation controls tumor-cell sensitivity to the proapoptotic ligand Apo2L/TRAIL. Nat Med 2007;13:1070-1077.

53 Clancy L, et al: Preligand assembly domainmediated ligand-independent association between TRAIL receptor 4 (TR4) and TR2 regulates TRAIL-induced apoptosis. Proc Natl Acad Sci USA 2005;102:18099-18104.

54 Xiang H, et al: Tissue distribution, stability, and pharmacokinetics of Apo2 ligand/tumor necrosis factor-related apoptosis-inducing ligand in human colon carcinoma COLO205 tumor-bearing nude mice. Drug Metab Dispos 2004;32:1230-1238.

55 Menon LG, Kelly K, Yang HW, et al: Human bone marrow-derived mesenchymal stromal cells expressing S-TRAIL as a cellular delivery vehicle for human glioma therapy. Stem Cells 2009;27:2320-2330.

56 Mohr A, Albarenque SM, Deedigan L, et al: Targeting of XIAP combined with systemic mesenchymal stem cell-mediated delivery of sTRAIL ligand inhibits metastatic growth of pancreatic carcinoma cells. Stem Cells 2010; 28:2109-2120.
57 Li HL, et al: Cleavage of BID by caspase 8 mediates the mitochondrial damage in the Fas pathway of apoptosis. Cell 1998;94:491-501.

58 Shamimi-Noori S, et al: Cisplatin enhances the antitumor effect of tumor necrosis factorrelated apoptosis-inducing ligand gene therapy via recruitment of the mitochondria-dependent death signaling pathway. Cancer Gene Ther 2008;15:356-370.

59 Sonnemann J, et al: Histone deacetylase inhibitors interact synergistically with tumor necrosis factor-related apoptosis-inducing ligand (TRAIL) to induce apoptosis in carcinoma cell lines. Invest New Drugs 2005;23: 99-109.

60 Su L, Liu G, Hao X, et al: Death receptor 5 and cellular FLICE-inhibitory protein regulate pemetrexed-induced apoptosis in human lung cancer cells. Eur J Cancer 2011;47:24712478.

61 Ding W, et al: Synergistic antitumor effect of TRAIL in combination with sunitinib in vitro and in vivo. Cancer Lett 2010;293:158-166.

62 Vaculova A, Kaminskyy V, Jalalvand E, et al: Doxorubicin and etoposide sensitize small cell lung carcinoma cells expressing caspase- 8 to TRAIL. Mol Cancer 2010;9:87.

63 Luster TA, Carrell JA, McCormick K, et al: Mapatumumab and lexatumumab induce apoptosis in TRAIL-R1 and TRAIL-R2 antibody-resistant NSCLC cell lines when treated in combination with bortezomib. Mol Cancer Ther 2009;8:292-302.

64 Ciavarella S, et al: In vitro anti-myeloma activity of TRAIL-expressing adipose-derived mesenchymal stem cells. Br J Haematol 2012; 157:586-598.

$65 \mathrm{Kim}$ DR, et al: Combination of vorinostat and adenovirus-TRAIL exhibits a synergistic antitumor effect by increasing transduction and transcription of TRAIL in lung cancer cells. Cancer Gene Ther 2011;18:467-477.

66 Kataoka T, et al: FLIP prevents apoptosis induced by death receptors but not by perforin/ granzyme $\mathrm{B}$, chemotherapeutic drugs, and gamma irradiation. J Immunol 1998; 161: 3936-3942

67 Okano H, et al: Cellular FLICE/caspase-8-inhibitory protein as a principal regulator of cell death and survival in human hepatocellular carcinoma. Lab Invest 2003;83:1033-1043.

68 Gill C, Dowling C, O'Neill AJ, et al: Effects of cIAP-1, cIAP-2 and XIAP triple knockdown on prostate cancer cell susceptibility to apoptosis, cell survival and proliferation. Mol Cancer 2009;8:39.

69 Albarenque SM, et al: RNAi-mediated inhibition of XIAP combined with systemic mesenchymal stem cell-mediated delivery of sTRAIL inhibits metastatic growth of pancreatic carcinoma cells. J Comp Pathol 2010;143: 330-330.

70 Stadel D, et al: TRAIL-induced apoptosis is preferentially mediated via TRAIL Receptor 1 in pancreatic carcinoma cells and profoundly enhanced by XIAP Inhibitors. Clin Cancer Res 2010;16:5734-5749. 
-71 Wu X-X, et al: Low concentrations of doxorubicin sensitizes human solid cancer cells to tumor necrosis factor-related apoptosis-inducing ligand (TRAIL)-receptor (R) 2-mediated apoptosis by inducing TRAIL-R2 expression. Cancer Sci 2007;98:1969-1976.

-72 Glennie S, et al: Bone marrow mesenchymal stem cells induce division arrest anergy of activated T cells. Blood 2005;105:2821-2827.

73 Corcione A, et al: Human mesenchymal stem cells modulate B-cell functions. Blood 2006; 107:367-372.

74 Ramasamy R, Fazekasova H, Lam EWF, et al: Mesenchymal stem cells inhibit dendritic cell differentiation and function by preventing entry into the cell cycle. Transplantation 2007;83:71-76.

-75 LeBlanc K, et al: Mesenchymal stem cells for treatment of steroid-resistant, severe, acute graft-versus-host disease: a phase II study. Lancet 2008;371:1579-1586.

76 Assmus B, et al: Transcoronary transplantation of progenitor cells after myocardial infarction. N Engl J Med 2006;355:1222-1232.

-77 Schaechinger V, et al: Intracoronary bone marrow-derived progenitor cells in acute myocardial infarction. N Engl J Med 2006; 355:1210-1221.

-78 Hare JM, Chaparro SV: Cardiac regeneration and stem cell therapy. Curr Opin Organ Transplant 2008;13:536-542.

79 Lunde $\mathrm{K}$, et al: Intracoronary injection of mononuclear bone marrow cells in acute myocardial infarction. N Engl J Med 2006; 355:1199-1209.
80 Ortiz LA, et al: Mesenchymal stem cell en graftment in lung is enhanced in response to bleomycin exposure and ameliorates its fibrotic effects. Proc Natl Acad Sci USA 2003; 100:8407-8411.

81 Islam MN, et al: Mitochondrial transfer from bone-marrow-derived stromal cells to pulmonary alveoli protects against acute lung injury. Nat Med 2012;18:759-765.

82 Nakamura K, et al: Antitumor effect of genetically engineered mesenchymal stem cells in a rat glioma model. Gene Ther 2004;11:11551164.

83 Sun B, Roh KH, Park JR, et al: Therapeutic potential of mesenchymal stromal cells in a mouse breast cancer metastasis model. Cytotherapy 2009;11:289-298.

84 Qiao L, Zhao TJ, Wang FZ, et al: NF-kappaB downregulation may be involved the depression of tumor cell proliferation mediated by human mesenchymal stem cells. Acta Pharmacol Sin 2008;29:333-340.

85 Djouad F, et al: Immunosuppressive effect of mesenchymal stem cells favors tumor growth in allogeneic animals. Blood 2003;102:38373844 .

86 Karnoub AE, Dash AB, Vo AP, et al: Mesenchymal stem cells within tumour stroma promote breast cancer metastasis. Nature 2007; 449:557-563.

87 Sasser AK, et al: Interleukin-6 is a potent growth factor for ER-alpha-positive human breast cancer. FASEB J 2007;21:3763-3770.
88 Sasser AK, et al: Human bone marrow stromal cells enhance breast cancer cell growth rates in a cell line-dependent manner when evaluated in 3D tumor environments. Cancer Lett 2007;254:255-264.

89 Sanchez CG, et al: Activation of autophagy in mesenchymal stem cells provides tumor stromal support. Carcinogenesis 2011;32:964972.

90 Rubio D, Garcia-Castro J, Martin MC, et al: Spontaneous human adult stem cell transformation. Cancer Res 2005;65:3035-3039, 4969.

91 Wang Y, et al: Outgrowth of a transformed cell population derived from normal human BM mesenchymal stem cell culture. Cytotherapy 2005;7:509-519.

92 Aguilar S, et al: Murine but not human mesenchymal stem cells generate osteosarcomalike lesions in the lung. Stem Cells 2007;25: 1586-1594.

93 Houghton J, et al: Gastric cancer originating from bone marrow-derived cells. Science 2004;306:1568-1571.

94 Bernardo ME, et al: Human bone marrowderived mesenchymal stem cells do not undergo transformation after long-term in vitro culture and do not exhibit telomere maintenance mechanisms. Blood 2007;110:367A.

95 clinicaltrials.gov. Clinical trials related to search term Mesenchymal stem cells. 2013. http://clinicaltrials.gov/ct2/results?term= mesenchymal+stem+cells. 